\title{
Strain hardening in 2D discrete dislocation dynamics simulations: a new '2.5D' algorithm
}

\author{
S. M. Keralavarma ${ }^{1,2}$, W. A. Curtin ${ }^{2}$ \\ ${ }^{1}$ Department of Aerospace Engineering, Indian Institute of Technology Madras \\ Chennai 600036, India \\ ${ }^{2}$ Institute of Mechanical Engineering, Ecole Polytechnique Federale de Lausanne \\ CH-1015 Lausanne, Switzerland
}

\begin{abstract}
The two-dimensional discrete dislocation dynamics (2D DD) method, consisting of parallel straight edge dislocations gliding on independent slip systems in a plane strain model of a crystal, is often used to study complicated boundary value problems in crystal plasticity. However, the absence of truly three dimensional mechanisms such as junction formation means that forest hardening cannot be modeled, unless additional so-called '2.5D' constitutive rules are prescribed for short-range dislocation interactions. Here, results from three dimensional dislocation dynamics (3D DD) simulations in an FCC material are used to define new constitutive rules for short-range interactions and junction formation between dislocations on intersecting slip systems in 2D. The mutual strengthening effect of junctions on preexisting obstacles, such as precipitates or grain boundaries, is also accounted for in the model. The new ' $2.5 \mathrm{D}$ ' DD model, with no arbitrary adjustable parameters beyond those obtained from lower scale simulation methods, is shown to predict athermal hardening rates, differences in flow behavior for single and multiple slip, and latent hardening ratios. All these phenomena are well-established in the plasticity of crystals and quantitative results predicted by the model are in good agreement with experimental observations.
\end{abstract}

Keywords: A. dislocations; A. strengthening mechanisms; B. crystal plasticity; B. constitutive behaviour; C. numerical algorithms

\section{Introduction}

Dislocations are line defects in a crystal lattice whose properties and interactions with other defects control macroscopic mechanical properties such as strength, work hardening and ductility. Glide of dislocations in response to applied shear stresses cause plastic deformation while the stress required to sustain plastic flow is governed by the properties of obstacles to dislocation glide such as forest dislocations, precipitates, grain boundaries, dislocation cell walls, etc. The flow stress $\tau$ is typically inversely related to the obstacle spacing $d$, i.e. $\tau \propto d^{-n}$, with the exponent $n$ dependent on several factors such as the type, strength and density of the obstacles and with $d$ ranging from $10 \mathrm{~nm}<d<10$ $\mu \mathrm{m}$. Continuum models of plasticity embody these strengthening mechanisms implicitly, 
but are mainly based on experimental observations at macroscopic length scales $(>1$ $\mathrm{mm}$ ). It is now experimentally well-established (Fleck and Hutchinson, 1997; Arzt, 1998; Uchic et al., 2009), that plasticity is size-dependent when specimen dimensions or spatial variations in stress become comparable to or smaller than the microstructural length scales, with increasing strength with reduced sample dimensions or increasing stress or strain gradients. Well known limitations of continuum plasticity theory, such as the mesh dependence of results in strain localization calculations (De Borst et al., 1993) and the inability to predict realistic values for the fracture toughness of ductile materials (Tvergaard and Hutchinson, 1992), are probably related to length scale effects due to the inherently discrete nature of plasticity in such situations.

Modeling size-dependent behavior requires augmenting continuum plasticity models with embedded length scales. Top-down approaches involve the introduction of gradient plasticity models (Fleck et al., 1994; Gao et al., 1999; Chakravarthy and Curtin, 2011b), while bottom-up methods directly model the dislocations and their motion. The main advantage of the latter discrete dislocation (DD) modeling is the absence of constitutive assumptions at the macro scale, which allows for size-dependent behavior to emerge naturally. The DD method treats dislocations as discrete line defects embedded in an elastic continuum, which move and interact with other crystal defects in response to their mutual interaction forces and the applied mechanical fields, and plastic deformation results from the collective motion of the dislocations. Fully three-dimensional models (3D DD) consider a 3D simulation cell containing closed dislocation loops that nucleate from Frank-Read sources and glide and interact with other defects in response to applied loads. Realistic representation of the crystallographic slip systems and the geometry of the dislocation loops on individual slip systems naturally leads to the emergence of forest hardening by mutual entanglement of these loops. However, 3D DD is computationally very expensive, particularly for problems with non-homogeneous loading and explicit interfaces. An alternative approach is thus a two-dimensional method (2D DD) that sacrifices some physical fidelity for computational efficiency. Following early work by Amodeo and Ghoniem (1990), the most commonly used 2D DD model by Van der Giessen and Needleman (1995) considers parallel straight edge dislocations in plane strain, which permits the study of problems involving complex inhomogeneous loading such as the growth of cracks (Deshpande et al., 2001; Chakravarthy and Curtin, 2010b), bi-material fracture (O'Day and Curtin, 2005), micro-void evolution (Segurado and Llorca, 2009), indentation (Widjaja et al., 2007), thin films (Nicola et al., 2006; Chng et al., 2006) and sliding/friction (Deshpande et al., 2004). The 2D DD method captures the long range interactions between dislocations, which enables size and gradient effects to emerge naturally, but truly 3D phenomena like junction formation and forest hardening are missing, so that standard 2D DD models predict elastic/perfectly-plastic response under nominally homogeneous loading conditions. The objective of the present paper is to propose a new algorithm for incorporating strain hardening into 2D DD simulations, which improves upon existing approaches in the literature in several important ways.

Since 2D DD lacks the physics of junction formation, constitutive rules need to be prescribed to represent the effects of the corresponding phenomena in 3D. Such efforts are known as '2.5D' DD (Benzerga et al., 2004; Gómez-Garcia et al., 2006). The first 
such model was proposed by Benzerga et al. (2004), in which dislocations on intersecting slip planes are allowed to form 'junctions' when they approach within a critical interaction distance, which was fixed at six times the magnitude of the Burgers vector. These immobile junctions, which act as obstacles to slip and possibly also anchoring points for new 'dynamic' Frank-Read sources, can be destroyed by sufficiently high resolved shear stresses on the participating dislocations. The resulting evolution of the source and obstacle population was shown to predict strain hardening as well as size effects in micron-sized compression specimens (Benzerga et al., 2004; Guruprasad and Benzerga, 2008). An alternative approach adopted by Gómez-Garcia et al. (2006) considered a single crystal undergoing nominally homogeneous deformation, a cross-section plane of which was analyzed using periodic boundary conditions to simulate bulk behavior. In contrast with the approach of Benzerga et al. (2004), their model accounted for dislocation density multiplication due to the 3D flux of expanding dislocation loops crossing the plane of analysis, as opposed to nucleation of dipoles from fixed sources. These dislocations subsequently formed junctions with dislocations on other slip systems according to a critical distance criterion, where the interaction distance was taken to vary inversely as the square root of the local dislocation density, consistent with the observation that the lengths of junction segments in 3D satisfy the above scaling relation. Despite these differences, strain hardening in both these models resulted from the addition of junction obstacles, following a critical interaction distance criterion in the plane of analysis. However, if we view 2.5D DD as a projection of 3D DD onto a 2D plane, then dislocations on intersecting slip planes in $3 \mathrm{D}$ can intersect and form junctions at any location in the $2 \mathrm{D}$ plane, with consequent strengthening of all dislocations in the vicinity of the junction. This cannot be captured using a critical distance criterion for junction formation in 2D. Moreover, the existing 2.5D DD models introduce several constitutive parameters related to the strength and stability of the junctions that are used as adjustable parameters to obtain a desired hardening rate. Thus, there is some need for a more physically-based 2.5D DD model that cleanly represents realistic 3D phenomena with few additional assumptions or inputs.

In this paper, a new 2.5D DD model is proposed based on the ideas that (i) constitutive rules for short range dislocation interactions in $2 \mathrm{D}$ must reproduce the effects of the corresponding phenomena in $3 \mathrm{D}$ on the overall plasticity rather than attempt to reproduce the details of the mechanisms themselves, and (ii) constitutive parameters related to junction formation and other hardening phenomena must be derived from 3D DD simulations. We present such a physically-motivated 2.5D DD model using information from 3D DD to select key parameters. We then demonstrate that the model produces initial strengthening, due to preexisting glide obstacles, followed by linear hardening due to forest interactions, with hardening rates under single and multiple slip conditions consistent with experimental behavior. This 2.5D DD model thus represents a general method to include multiple strengthening mechanisms, hardening, and multi-axial plastic behavior, into plasticity simulations of problems involving complex loading conditions in materials with realistic deformation behavior.

The remainder of the paper is organized as follows. A brief summary of the standard 2D DD simulation method is provided in Section 2.1, followed by a detailed presentation of the new 2.5D algorithm in Section 2.2. The method is illustrated by studying the response 
of bulk single crystals using periodic unit cells subjected to arbitrary plane strain loading. Details of the geometry, boundary conditions and the solution procedure are explained in Section 3. The corresponding simulation results are presented in Section 4, followed by a discussion of the results in Section 5 and conclusions in Section 6 .

\section{2.5D Dislocation Dynamics}

\subsection{D DD Constitutive Rules}

Before introducing additional constitutive rules for our 2.5D DD model, we briefly summarize the general framework and constitutive rules for the standard 2D DD model. The 2D DD model of Van der Giessen and Needleman (1995) considers a plane strain model of a crystal containing parallel straight dislocations normal to the plane of analysis and subjected to arbitrary in plane loading. Only pure edge dislocations are considered due to the fact that the Volterra solution for a straight edge dislocation in an infinite medium satisfies plane strain conditions. The edge dislocations exist on pre-determined glide planes in the crystal. The Peach-Koehler force on an individual dislocation has contributions from the stress fields generated by all the other dislocations in the system when treated as an infinite body, additional image stresses of all the dislocations due to the imposed boundary conditions in a real finite body, and the applied loading on that finite body. For a dislocation $i$ of Burgers vector $\mathbf{b}^{i}$ lying on a slip plane with normal vector $\mathbf{n}^{i}$, the Peach-Koehler glide force is given as

$$
f_{g}^{i}=\mathbf{b}^{i} \cdot\left(\hat{\boldsymbol{\sigma}}+\sum_{j \neq i} \boldsymbol{\sigma}^{j}\right) \cdot \mathbf{n}^{i}
$$

where the $\hat{\boldsymbol{\sigma}}$ field includes all the applied and image forces (computed as detailed later) and the direct interaction of dislocation $j$ is $\boldsymbol{\sigma}^{j}$.

Nucleation, glide, pinning, and unpinning of dislocations is governed by a set of constitutive rules based on lower-level physics, as follows. A set of discrete points represent Frank-Read sources in 3D. Each source can nucleate a dislocation dipole when the resolved shear stress on the source exceeds the critical nucleation stress of the source $\tau_{\text {nuc }}$ over a critical nucleation time $t_{\text {nuc }}$, mimicking the bow out process of a Frank-Read source projected onto the $2 \mathrm{D}$ plane. A statistical distribution of the source strengths following a normal distribution with average source strength $\bar{\tau}_{\text {nuc }}$ and standard deviation $\tau_{\text {sd }}$ is assumed, since the nucleation strength of a Frank-Read source in 3D depends on the length of the source, which is not represented in $2 \mathrm{D}$. The glide velocity $v_{g}^{i}$ of nucleated dislocation $i$ is given by a linear viscous mobility law as

$$
f_{g}^{i}=B_{g} v_{g}^{i}
$$

where $B_{g}$ is the phonon drag coefficient that can be computed atomistically (Olmsted et al., 2005). The Peierls barrier for glide is usually neglected, which is reasonable for edge dislocations in FCC metals, but can be included when dominant (Olarnrithinun et al., 2013). 
The simulation domain also contains discrete point obstacles randomly distributed on the slip planes with a specified average density. A dislocation encountering an obstacle in its glide path is pinned at the obstacle until the magnitude of the resolved shear stress on the pinned dislocation reaches the pinning strength $\tau_{\text {obs }}$ of the obstacle, at which point the dislocation is released from the obstacle and resumes glide in the direction of the Peach-Koehler force. Chakravarthy and Curtin (2010a) have shown that the resolved shear stress for macroscopic yielding on a slip system in the $2 \mathrm{D}$ model depends on the source and obstacle strengths and obstacle spacing $L_{\text {obs }}$ as

$$
\tau_{y}=\sqrt{\frac{4 \mu b}{\pi(1-\nu)} \frac{\tau_{\mathrm{obs}}}{5.7 L_{\mathrm{obs}}}+\tau_{s}^{2}}
$$

where $\tau_{s}=\bar{\tau}_{\text {nuc }}-2 \tau_{\text {sd }}$. In $2 \mathrm{D}$, a discrete set of slip planes with spacing $d$ is used, and so the average obstacle spacing is $L_{\mathrm{obs}}=N_{\mathrm{s}} /\left(\rho_{\mathrm{obs}} d\right)$ where $N_{\mathrm{s}}$ is the number of independent slip systems and $\rho_{\text {obs }}$ is the imposed 2D obstacle density. Finally, dislocations of opposite sign on the same slip plane annihilate each other when they are within a critical annihilation distance $L_{\mathrm{ann}}=6 b$.

\subsection{5D DD Constitutive Rules for Strain Hardening}

The flow stress in a plastically deforming crystal is controlled by the short range interactions of glissile dislocations with obstacles to glide such as forest dislocations, precipitates, grain boundaries, etc. Dislocations can move past an array of localized obstacles by bowing out between the obstacles until a sufficient force is generated to pull the pinned dislocation segments past the obstacle (or loop around it). The stress required to overcome an array of obstacles is well-known to scale inversely with the average obstacle spacing (Hirth and Lothe, 1968). Dislocations also multiply with increasing plastic strain so that the average spacing between forest dislocations scales as the inverse square root of the dislocation density. Hence, the flow stress for a plastically deforming crystal subject only to forest hardening scales with the square root of the dislocation density. In the standard 2D DD model, the population of obstacles is fixed a priori, thus representing precipitates or grain boundaries, and the obstacle strength $\tau_{\text {obs }}$ and spacing $L_{\text {obs }}$ are chosen to achieve the desired yield stress, according to Eq.(3) above, associated with a real 3D distribution of such obstacles. The material response is then elastic/perfectly-plastic. However, to represent forest hardening, a model must account for an evolving population of forest obstacles that operate together with the fixed obstacle to impede dislocation motion.

As noted earlier, previous 2.5D DD models by Benzerga et al. (2004) and GómezGarcia et al. (2006) assumed that a forest junction is formed between two dislocations on intersecting slip planes when they fall within a given radius centered at the intersection point of the slip planes. Benzerga et al. (2004) assumed this radius to be fixed at $6 b$ while Gómez-Garcia et al. (2006) took the capture radius to evolve inversely with the square root of the dislocation density, thus capturing some 3D effect. Upon formation, these new $2 \mathrm{D}$ junctions act as additional obstacles with strength proportional to $\mu b / L_{l}$, where $L_{l}$ is a local length that signifies the size of the junction segment. The junctions impede further glide on both slip planes until the resolved shear stress on either dislocation exceeds the junction strength, at which point the junction is destroyed and glide 
resumes on the two slip planes. These junction obstacles are thus treated differently than, and independently of, the initial fixed obstacles. Here, we consider an obstacle in a 2D simulation to represent a projection of an array of obstacles in the third dimension, so that their density and strength in 2D is representative of the actual obstacle density in 3D. Thus, a "junction" obstacle in a 2D simulation represents an array of junctions in the third dimension corresponding to some local density of forest dislocations, and any real 3D dislocation moves through a field of both fixed and "junction" obstacles. Furthermore, such a junction obstacle need not occur at the intersection point of two 2D slip planes nor rely on the presence of any actual dislocation at the $2 \mathrm{D}$ point of the junction obstacle.

3D DD simulations show that the important microscopic parameters relevant to the evolution of forest hardening are the forest dislocation density and the mean free path of glissile dislocations (Devincre et al., 2008; Kubin et al., 2008). Specifically, the junction strength $\tau_{\text {jcn }}^{i}$ for a junction on slip system $i$ is the resolved shear stress required for a dislocation to cut through an array of junctions with the dislocation forest. This is related to the local average spacing $L_{f}^{i}$ between forest dislocations on the remaining slip systems as

$$
\tau_{\mathrm{jcn}}^{i}=\alpha \frac{\mu b}{L_{f}^{i}}
$$

where $\mu$ is the shear modulus, $b$ is the modulus of the Burgers vector and $\alpha$ is a constant, which in simple models averaged over various junction types in an FCC material is $\alpha \approx 0.5$. $L_{f}^{i}$ is related to the local forest density, $\rho_{f}^{i}$, as $L_{f}^{i} \approx 1 / \sqrt{\rho_{f}^{i}}$, where the neighborhood defining "local" is specified later.

The mean free path $L^{i}$ on slip system $i$ is the average glide distance of a mobile dislocation before it is halted ("stored") by interaction with the forest of dislocations on other slip systems. The mean free path determines the plastic strain increment for each dislocation glide step, and so an increasing dislocation density causes not only an increase in strength but also a decrease in mean free path (plastic strain increment). Therefore the strain hardening rate is inversely related to the mean free path. Kubin and co-workers (Devincre et al., 2008; Kubin et al., 2008) have used 3D DD to estimate the mean free path for slip systems in an FCC crystal. They show that

$$
L^{i}=\frac{K}{\sqrt{\rho_{f}^{i}}}=K L_{f}^{i}
$$

where $K$ is a constant that depends on loading orientation. Their reported value of $K$ was a decreasing function of the number of active slip systems with $K \sim 10$ for a double slip orientation to $\sim 5$ for four active slip systems. Here, we choose a constant value for the parameter $K$ irrespective of the loading orientation, which is reasonable given the 2D idealization and the fact that the first order variation in $L^{i}$ results from the variation of the mean forest spacing $L_{f}^{i}$ as a function of the loading orientation. The relationship between the mean free path $L^{i}$ and the mean spacing between forest dislocations $L_{f}^{i}$ is illustrated in Fig. 1(a), which shows the dislocation on slip system $i$ gliding through a random field of forest dislocations on its slip plane. In a 2D simulation, where the plane of analysis is perpendicular to the slip plane, new junction obstacles should be introduced 
(a)

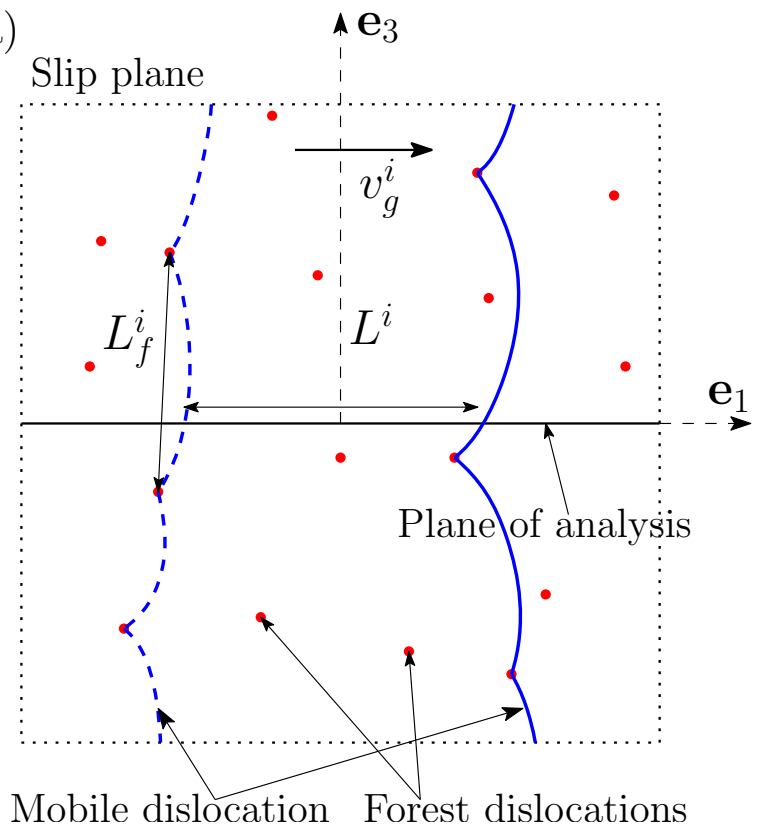

(b)

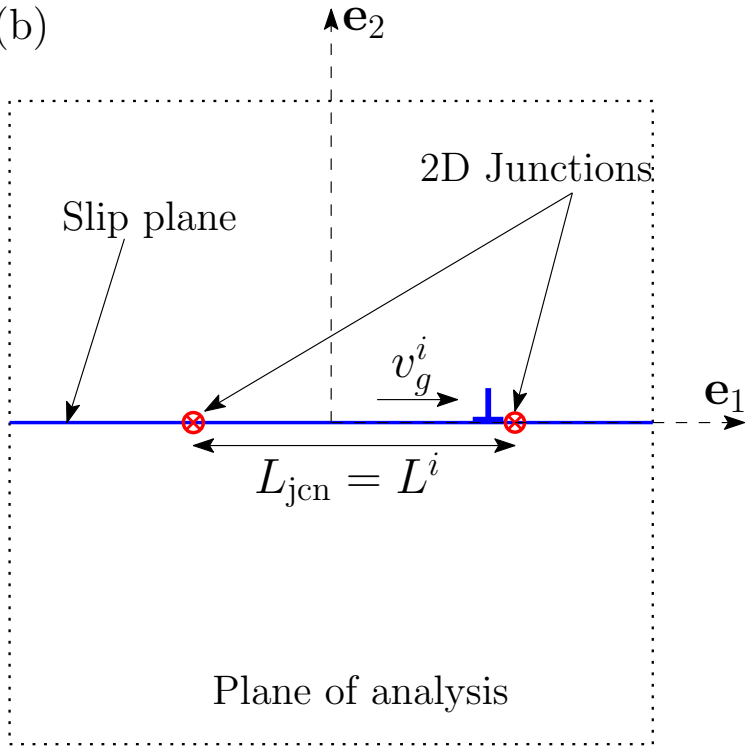

Figure 1: Schematic diagram showing (a) the mean free path $L^{i}$ of a dislocation gliding through a random array of forest dislocations on its slip plane and (b) the corresponding spacing of junction obstacles in the $2 \mathrm{D}$ plane of analysis.

with a mean spacing $L_{\mathrm{jcn}}$ approximately equal to the mean free path $L^{i}$ (see Fig. 1b), and not the average forest dislocation spacing $L_{f}^{i}$. This choice of the junction spacing accounts for the fact that not all forest dislocations form junctions with a mobile dislocation.

In addition to the junction obstacles, the simulation domain has a fixed population of preexisting obstacles with initial strengths set according to the yield stress of the material. Consideration of these non-forest obstacles is important for the modeling of real material microstructures where a significant part of the strength results from engineered defects initially present in the material, such as solute atoms, precipitates or phase boundaries. These obstacles account for the initial yield strength of the material, as well as affect the subsequent flow strength due to the simultaneous interaction of a glide dislocation with an array of fixed obstacles and forest junctions. Line-tension simulations of a long dislocation line moving through a random array of two types of obstacles (Dong et al., 2010) provide useful insight into this problem. Consider two types of obstacles $i=1,2$ with obstacle strengths $\tau_{\mathrm{o}_{i}}$ and obstacle densities $\rho_{i}$. Let $\tau_{i}$ denote the average flow strength due to each type of obstacle acting alone. When both obstacle types are present at comparable densities (within a factor of 50 in density, encompassing many cases of interest (Dong et al., 2010)), the overall strength is given by

$$
\tau_{\text {tot }}^{2}=\tau_{1}^{2}+\tau_{2}^{2}
$$

This result was obtained long ago (Foreman and Makin, 1967; Brown and Ham, 1971) but without a clear delineation of the regime of obstacle densities over which the result is accurate. In order to achieve realistic strain hardening rates in a 2D DD simulation, it is necessary to introduce new junction obstacles with strengths and mean spacing on the 


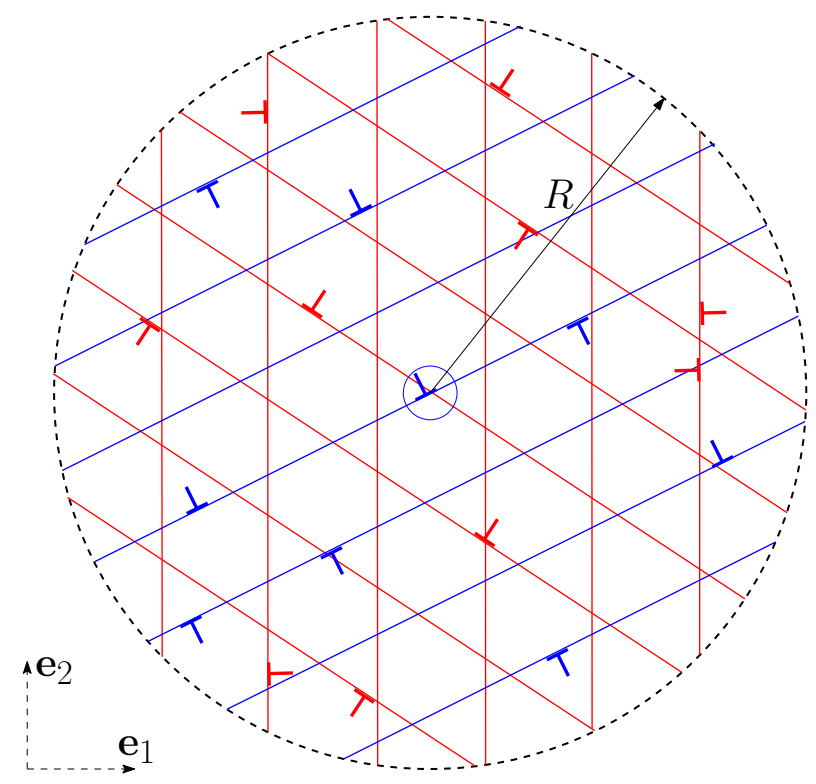

Figure 2: Sampling neighborhood for introduction of new junction obstacles in the 2.5D algorithm. A random dislocation and glide plane are chosen (as indicated) for evaluation as a possible junction location. Surrounding dislocations within a radius $R$ are computed for both the glide plane dislocations (blue) and the forest dislocations (red) on other slip systems.

slip planes in accordance with equations (4) and (5) and, furthermore, to strengthen all other forest and fixed obstacles in the vicinity according to Eq.(6). All of these features evolve locally according to the local dislocation densities on the various slip systems. The algorithm for implementing these features in a 2.5D DD simulation is described next.

At the end of each simulation time step, the material contains a spatial distribution of dislocations, forest/junction obstacles, and pre-existing fixed obstacles lying on the various discrete slip planes in the system. A point is picked at random along one slip plane $i$ in the system as a potential position of a new junction obstacle on that slip plane. In practice, and with no loss of generality, we choose this point to coincide with an existing dislocation, since this restricts the sampling of possible positions of junctions to regions where dislocations actually exist in the sample. The neighborhood of the chosen point on a slip system $i$ is then examined to determine (i) the current density of junction obstacles on slip system $i$ and (ii) the density of forest dislocations on all intersecting slip systems, as shown schematically in Fig. 2. Specifically, a circular domain of radius $R(N)$ around the chosen point and containing $N$ dislocations, irrespective of slip system, is constructed. Within this radius, the number of existing junctions $N_{\mathrm{jcn}}^{i}$ and the number of forest dislocations $N_{f}^{i}$ for each slip system $i$ are measured. The average spacing of the existing junctions on slip system $i$ is computed as

$$
L_{\mathrm{jcn}}^{i}=\frac{1}{\rho_{\mathrm{jcn}}^{i} d}, \quad \rho_{\mathrm{jcn}}^{i}=\frac{N_{\mathrm{jcn}}^{i}}{\pi R^{2}}
$$

where we are careful to account for the discrete slip plane spacing $d$ in the 2D problem. The mean free path of mobile dislocations on slip system $i$ is then computed using Eq.(5), 
with $\rho_{f}^{i}=\frac{N_{f}^{i}}{\pi R^{2}}$. If the existing junction spacing $L_{\mathrm{jcn}}^{i}$ on slip system $i$ is greater than the expected mean free path $L^{i}$, then a new junction obstacle is created at the location of the chosen dislocation. Finally, the new and old obstacle strengths within the radius $R$ are modified to account for the increased density of forest obstacle. The strength of the newly-created junction obstacle $\tau_{\text {jcn }}^{i}$ is given by Eq.(4) with $L_{f}^{i}=\left(\rho_{f}^{i}\right)^{-\frac{1}{2}}$. The strengths of all other junction obstacles on slip system $i$ within the radius $R$ are also increased to $\tau_{\text {jcn }}^{i}$, unless they are already larger than this value. And, the strengths of all the fixed obstacles within $R$ are increased according to Eq.(6),

$$
\tau_{\mathrm{obs}}^{i \quad 2}=\tau_{\mathrm{obs}}^{2}+\tau_{\mathrm{jcn}}^{i 2}
$$

unless they are already larger than this value. This procedure accounts for the fact that the strengthening is due to an interaction of a dislocation with the combined array of fixed and forest obstacles as it glides.

The overall algorithm above must be repeated on each slip system. The algorithm must also be performed with sufficient frequency such that the junction density can keep up with the spatially-evolving dislocation density in the specimen. However, since introduction of a new junction only occurs if warranted by the current forest densities and current junction densities, more-frequent insertion attempts do not lead to an increasing number of junctions; the junction creation rate is thus well defined. The junction density in our algorithm is currently a monotonically increasing function of the plastic strain; i.e. recovery mechanisms (usually thermally activated) that can potentially lead to a decrease in the local junction density are not modeled. While real 3D junctions can be destroyed by the application of sufficiently high stresses, the evolution of the mean free path in 3D is expected to account for the net effect of the junction formation and destruction processes. Hence the algorithm may be expected to yield reasonable predictions for the hardening rates at low temperatures, so long as the correct estimate for the mean free path as a function of plastic strain is used. One might imagine that the algorithm depends on the number $N$ of dislocations that determines the radius $R(N)$. However, while $N$ should be large enough to measure a meaningful density yet small enough that local fluctuations in dislocation density are not smoothened out, we show below that results for the strain hardening are insensitive to $N$ over the range $N 10-100$. Obviously, the radius $R$ of the sampling neighborhood decreases as the overall dislocation density increases, and so the algorithm does have an evolving internal length scale that controls the hardening process. However, this is physical since the 3D processes scale with local forest density. Finally, when parts of the circle of radius $R$ fall outside the sample, only the area in the domain of analysis is used when calculating densities.

The key difference between the new model described above and the earlier models of Benzerga et al. (2004); Gómez-Garcia et al. (2006) is in the prescription to add new junction obstacles in accordance with the evolving local mean free path for glide on each slip system as a function of the forest dislocation density rather than a critical distance criterion tied to the intersection points of the slip traces in the $2 \mathrm{D}$ plane of analysis. The dislocation nucleation criterion in the model of Gómez-Garcia et al. (2006) assumed a constant value for the mean free path, while its effect on the density of junction obstacles 
was indirectly accounted for by making the critical distance for junction formation a decreasing function of the dislocation density. However, it was unclear how the above rules would lead to the correct evolution of the junction densities as the dislocation density increases. Also, unlike in the previous models, the combined effect of forest dislocations and pre-existing non-forest obstacles on strengthening are accounted for using the results of previous studies on dislocation glide though two populations of obstacles using the line tension model. This is important for the study of alloy materials where a significant part of the strengthening results from pre-existing obstacles such as precipitates. Gómez-Garcia et al. (2006) assumed a pure material with no pre-existing obstacles to glide while the model of Benzerga et al. (2004) assumed a fixed initial density of point obstacles, although their strength was assumed to be independent of the density of forest dislocations.

\section{Test Geometry and Implementation Details}

We simulate the stress-strain response of a bulk crystal using a periodic representative volume element (RVE) to avoid any finite-size or surface effects, using a formulation for periodic boundary conditions previously employed by Hussein et al. (2008). The sample is taken as a rectangular domain $V$ of dimensions $L \times H$ in the $x_{1}-x_{2}$ plane containing discrete edge dislocations on three slip systems as shown in Fig. 3(a). The three slip systems are symmetrically oriented such that the angle between neighboring slip systems is $60^{\circ}$; a symmetric orientation of the unit cell is chosen as shown in Fig. 3(a) with $\varphi=30^{\circ}$. The edge dislocations have their line direction along the $x_{3}$ axis and hence appear as discrete points in the plane of analysis.

Let $\boldsymbol{\sigma}, \boldsymbol{\epsilon}$ and $\mathbf{u}$ denote the stress, elastic strain and displacement fields in the crystal, which are doubly periodic with periods $L$ and $H$ in the $x_{1}$ and $x_{2}$ directions, respectively. Using the superposition method associated with linear elasticity, the fields are written as the sum of two constituent fields

$$
\boldsymbol{\sigma}=\tilde{\boldsymbol{\sigma}}+\hat{\boldsymbol{\sigma}}, \quad \boldsymbol{\epsilon}=\tilde{\boldsymbol{\epsilon}}+\hat{\boldsymbol{\epsilon}}, \quad \mathbf{u}=\tilde{\mathbf{u}}+\hat{\mathbf{u}}
$$

The fields with overscript are themselves the superposition of the singular Volterra fields of all the dislocations within the domain $V$, i.e.

$$
\tilde{\boldsymbol{\sigma}}=\sum_{i=1}^{N_{\text {disl }}} \boldsymbol{\sigma}^{i}, \quad \tilde{\boldsymbol{\epsilon}}=\sum_{i=1}^{N_{\text {disl }}} \boldsymbol{\epsilon}^{i}, \quad \tilde{\mathbf{u}}=\sum_{i=1}^{N_{\text {disl }}} \mathbf{u}^{i},
$$

where the superscript $i$ denotes the Volterra solution for dislocation $i$ and $N_{\text {disl }}$ denotes the total number of dislocations in the cell. Consequently, the $\sim$ fields are non-periodic and the - fields in (9) are correction fields that enforce periodicity of the overall solution as well as impose the remotely applied loads on the unit cell. Here, we consider a strain controlled plasticity simulation so that the average total strain in the unit cell at any given time $t$ equals a prescribed strain $\overline{\boldsymbol{\epsilon}}(t)$; i.e.

$$
\langle\boldsymbol{\epsilon}\rangle_{V}=\langle\tilde{\boldsymbol{\epsilon}}+\hat{\boldsymbol{\epsilon}}\rangle_{V}=\overline{\boldsymbol{\epsilon}} \quad \text { at time } t
$$

where the notation $\langle\cdot\rangle_{V}$ denotes a volume average over $V$. 
(b)

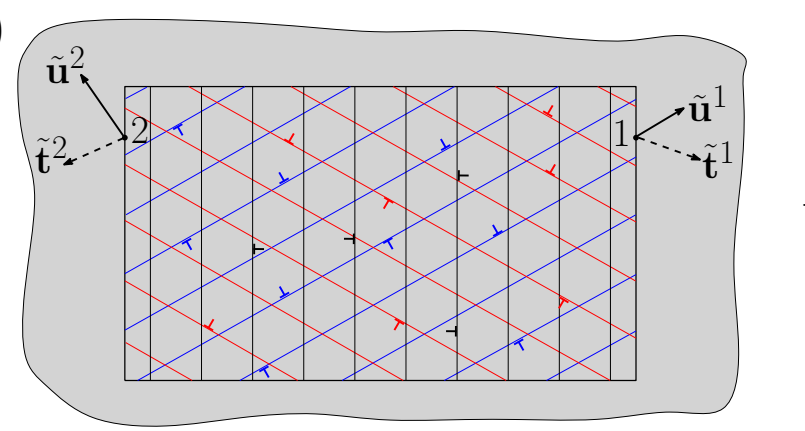

(a)

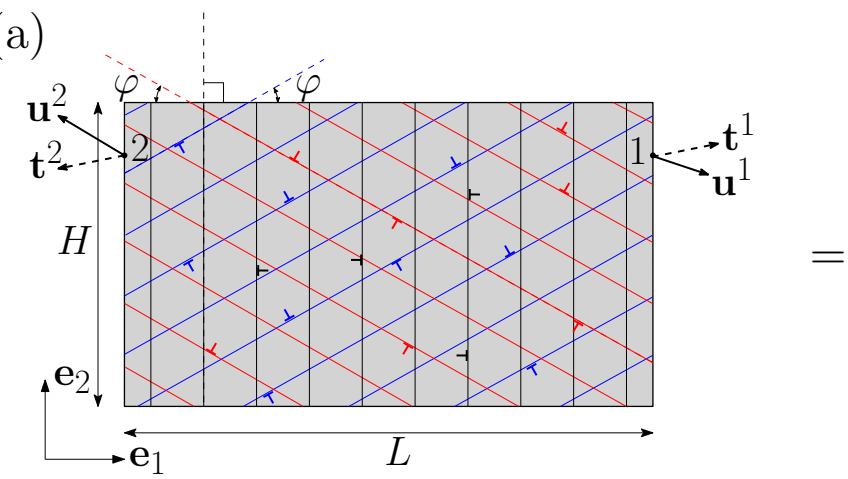

(c)

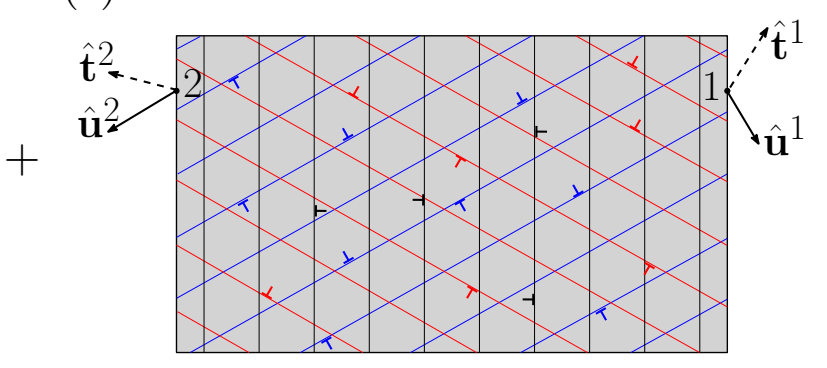

Figure 3: Superposition method for the solution of the elastic fields in a periodic unit cell.

The singularities and discontinuities in the stress and displacement fields are contained in the analytical fields so that the fields are smooth and can be calculated using the finite element method. Since the Volterra solution for the dislocations is self-equilibrating, the equilibrium equations for the ${ }^{-}$fields reduce to

$$
\nabla \cdot \hat{\boldsymbol{\sigma}}=\mathbf{0} \quad \text { in } V .
$$

We have

$$
\hat{\boldsymbol{\sigma}}=\mathbf{C}: \hat{\boldsymbol{\epsilon}}, \quad \hat{\boldsymbol{\epsilon}}=\frac{1}{2}\left(\nabla \hat{\mathbf{u}}+\nabla \hat{\mathbf{u}}^{T}\right)
$$

where $\mathbf{C}$ denotes the fourth order elastic stiffness tensor and $\hat{\mathbf{u}}$ is a continuous displacement field such that $\hat{\mathbf{u}}+\tilde{\mathbf{u}}-\overline{\boldsymbol{\epsilon}} \cdot \mathbf{x}$ is periodic, with $\mathbf{x}$ as the position vector in the RVE. Let the superscripts 1 and 2 denote the fields at two periodic image nodes 1 and 2 on the RVE boundary as shown in Fig. 3. We have from the periodicity of the overall displacement fields,

$$
\mathbf{u}^{2}-\mathbf{u}^{1}=\overline{\boldsymbol{\epsilon}} \cdot\left(\mathbf{x}^{2}-\mathbf{x}^{1}\right) \Longrightarrow \hat{\mathbf{u}}^{2}=\overline{\boldsymbol{\epsilon}} \cdot\left(\mathbf{x}^{2}-\mathbf{x}^{1}\right)-\left(\tilde{\mathbf{u}}^{2}-\tilde{\mathbf{u}}^{1}\right)+\hat{\mathbf{u}}^{1}
$$

Similarly, the total traction $\mathbf{t}=\boldsymbol{\sigma} \cdot \mathbf{n}$, where $\mathbf{n}$ is the outward unit normal on the RVE boundary, must be equal and opposite at image nodes 1 and 2 in order to satisfy periodicity, i.e.

$$
\mathbf{t}^{2}=-\mathbf{t}^{1} \Longrightarrow \hat{\mathbf{t}}^{2}=-\hat{\mathbf{t}}^{1}-\left(\tilde{\mathbf{t}}^{1}+\tilde{\mathbf{t}}^{2}\right)
$$

where $\tilde{\mathbf{t}}=\tilde{\boldsymbol{\sigma}} \cdot \mathbf{n}$ and $\hat{\mathbf{t}}=\hat{\boldsymbol{\sigma}} \cdot \mathbf{n}$ respectively. The governing Eqs.(12)-(13) for the fields subject to boundary conditions $(14)_{2}$ and $(15)_{2}$ are solved using the finite element method. Note that conditions (14) and (15) are imposed using multi-point constraints 
on the degrees of freedom on the periodic boundaries, which leads to an unsymmetric sparse stiffness matrix in the discretized finite element equations. The resulting equations are solved using the open source sparse solver LUSOL. Subsequently, the $\sim$ fields of the dislocations in the cell unit cell are superposed with the - fields to obtain the actual solution of the periodic boundary value problem, as illustrated in Fig. 3(a)-(c).

The simulation proceeds by applying the prescribed strain history $\overline{\boldsymbol{\epsilon}}(t)$ in discrete increments of time, $\delta t$. At each time step, the elastic fields in the specimen are computed using the superposition method described above, for the current positions of the dislocations. The resulting stress fields are used to compute the forces on the dislocations and their glide velocities through equation (2) and their positions are updated using a semi-implicit integration scheme for the equations of motion (Chakravarthy and Curtin, 2011a). The constitutive rules for short range interactions from section 2.1 are used to check for events such as nucleation from sources, dislocation annihilation and pinning and de-pinning at obstacles, while the introduction of new junctions and updating of existing obstacle and junction strengths follow the 2.5D DD algorithm in section 2.2. If the circle of radius $R(N)$, corresponding to the sampling neighborhood for junction formation in the 2.5D algorithm happens to fall partially outside the domain $V$, periodic images of the partial circles that overlap the domain are used to calculate densities. Dislocations crossing periodic boundaries are removed and reintroduced into the simulation cell on a matching slip plane from the opposite boundary in order to preserve the overall periodicity of slip in the crystal. Note that care must be taken to retain the displacement discontinuities due to slip at the periodic boundaries so as to avoid spurious singularities at the boundary. Also, perfect periodicity of slip requires that the cell dimensions and aspect ratios must be chosen such that $L$ is an integral multiple of $d / \sin \varphi$, where $d$ denotes the slip plane spacing and $H / L=\tan \varphi$ (see Fig. 3). Given the new configuration of dislocations in the cell at the end of the increment, the new stress fields are computed and the simulation proceeds in an iterative manner.

The unit-cell in Fig. 3(a) is deformed at a constant average strain rate $\dot{\bar{\epsilon}}$ in the $x_{1}-x_{2}$ plane. Purely deviatoric strain rates $\dot{\bar{\epsilon}}$ that satisfy plane strain conditions are considered such that one can write $\dot{\overline{\boldsymbol{\epsilon}}}=\dot{\bar{\epsilon}}_{11}\left(\mathbf{e}_{1} \otimes \mathbf{e}_{1}-\mathbf{e}_{2} \otimes \mathbf{e}_{2}\right)+\dot{\bar{\epsilon}}_{12}\left(\mathbf{e}_{1} \otimes \mathbf{e}_{2}+\mathbf{e}_{2} \otimes \mathbf{e}_{1}\right)$ in the coordinate system introduced in Fig. 3 , where the ratio $\dot{\bar{\epsilon}}_{12} / \dot{\bar{\epsilon}}_{11}$ can be varied to control the principal directions of the deformation. Since the crystal has $60^{\circ}$ rotation symmetry, a little consideration shows that there are six potential orientations of the principal axes of deformation, $30^{\circ}$ apart, which correspond to single slip and six orientations corresponding to symmetric double slip midway between the single slip orientations. Here we look at three different loading scenarios: (i) single slip $\left(\dot{\bar{\epsilon}}_{11}=0\right.$ ), (ii) symmetric double slip $\left(\dot{\bar{\epsilon}}_{12}=0\right)$ and (iii) asymmetric double slip $\left(\dot{\bar{\epsilon}}_{11}=\dot{\bar{\epsilon}}_{12}\right)$, which are equivalent (in terms of the magnitudes of the slip rates) to one of the principal directions of strain rate oriented at $15^{\circ}, 0^{\circ}$ and $7.5^{\circ}$ with respect to the $\mathbf{e}_{1}$ axis, respectively. The work quantity conjugate to the applied strain rate $\dot{\overline{\boldsymbol{\epsilon}}}$ is the average stress in the unit cell, defined as $\overline{\boldsymbol{\sigma}}=\langle\boldsymbol{\sigma}\rangle_{V}$, which can be conveniently calculated by integration of the tractions on the boundaries of the unit cell using the divergence theorem.

The average resolved shear stress $\tau$ on a given slip system is plotted as a function of the corresponding resolved shear strain $\gamma$. The resolved shear stress on slip system $k(k$ 
$=1-3)$, identified by the slip direction $\mathbf{m}^{(k)}$ and slip plane normal direction $\mathbf{n}^{(k)}$, is given by $\tau^{(k)}=\overline{\boldsymbol{\sigma}}:\left(\mathbf{m}^{(k)} \otimes \mathbf{n}^{(k)}\right)$, where $\mathbf{m}^{(k)} \otimes \mathbf{n}^{(k)}$ is the Schmid tensor for slip system $k$. The shear strain rates $\dot{\gamma}^{(k)}$ can be obtained from the solution of the equations

$$
\dot{\overline{\boldsymbol{\epsilon}}}=\frac{1}{2} \sum_{k} \dot{\gamma}^{(k)}\left(\mathbf{m}^{(k)} \otimes \mathbf{n}^{(k)}+\mathbf{n}^{(k)} \otimes \mathbf{m}^{(k)}\right)
$$

In two dimensions, the above considerations yield two independent linear equations and at least two independent slip systems are required for a solution to exist for an arbitrary prescribed strain rate $\dot{\overline{\boldsymbol{\epsilon}}}$. For crystals with redundant slip systems, as in the present case, the solution is non-unique and Taylor's minimum dissipation principle states that the two active slip systems will be chosen such that the overall plastic dissipation is minimized. For a crystal with identical slip systems, this translates to the condition that $\sum_{k}\left|\dot{\gamma}^{(k)}\right|$ is minimized for the true slip rates. We verify that the active slip systems in all our simulations satisfy the above condition, which is a confirmation of the well-known result that plastic flow is associative for a material that obeys the Schmid law.

Material properties of Aluminum are assumed with Young's modulus $E=70 \mathrm{GPa}$, Poisson's ratio $\nu=0.33$ and modulus of the Burgers vector $b=0.25 \mathrm{~nm}$ on all slip systems. The dislocation glide mobility is assumed to be $B_{g}=10^{-4} \mathrm{~Pa}$ s. The average nucleation strength of the Frank-Read sources is taken to be $\bar{\tau}_{\text {nuc }}=50 \mathrm{MPa}$, with a standard deviation of $\tau_{\text {sd }}=0.2 \bar{\tau}_{\text {nuc }}$. We use a constant $\tau_{\text {obs }}=150 \mathrm{MPa}$ and vary the preexisting obstacle density to achieve desired values of the initial yield strength according to Eq.(3). Additionally, some results are also shown for crystals containing no pre-existing obstacles. Subsequently, the flow stress evolves as a function of the accumulated strain due to the formation of additional junction obstacles according to the $2.5 \mathrm{D}$ algorithm. The relevant parameters for the 2.5D algorithm are the junction strength parameter $\alpha$ in Eq.(4) and the mean free path parameter $K$ in Eq.(5). We first assume $\alpha=0.75$ and $K=10$, which are within the known ranges of these parameters based on atomistic and 3D DD simulations (cf. section 2.2), and the effects of these parameters on the predicted behavior are then examined. A unit cell with $L=15 \mu \mathrm{m}$ and $H=L / \sqrt{3} \mu \mathrm{m}$ is used, and simulations at other cell sizes for some loading conditions below show that this size is sufficient to preclude possible cell size effects. Hence the results may be considered representative of an infinite bulk material.

\section{Results}

We consider loading conditions that induce single slip, symmetric double slip, and asymmetric double slip. The 2.5D DD model should reproduce the correct behavior in all of these situations, consistent with the behavior of real single crystals. Under single slip, the hardening rate is very low, due to the absence of forest dislocations. Under multiple slip, the hardening rate is much higher due to the evolution of forest hardening. The strain hardening rate in multiple slip is typically in the range $\mu / 200-\mu / 100$ for FCC materials (Argon, 2008; Kocks and Mecking, 2003). Note that no strain hardening is predicted in the absence of the $2.5 \mathrm{D}$ constitutive rules in any of the simulations reported 

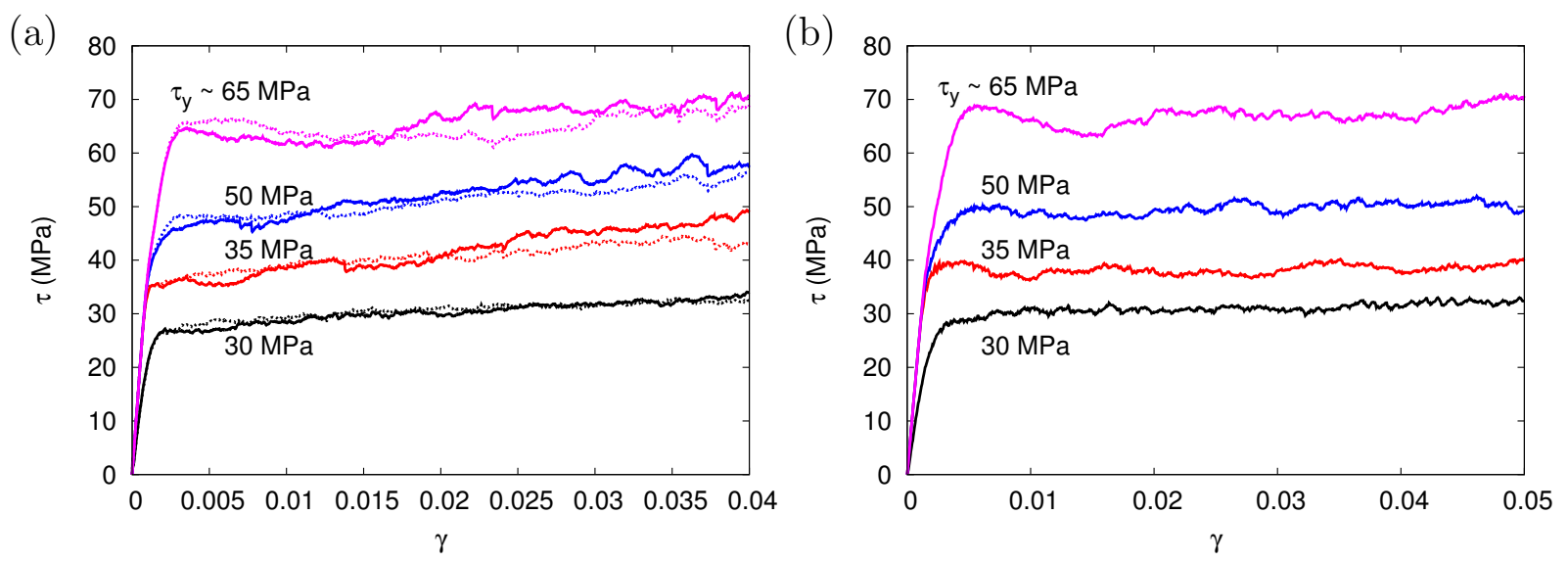

Figure 4: Stress-strain curves from the 2.5D DD simulations for single crystals of varying initial yield strengths $\tau_{y}$. (a) Symmetric double slip $\left(\dot{\bar{\epsilon}}_{11}=-\dot{\bar{\epsilon}}_{22}, \dot{\bar{\epsilon}}_{12}=0\right)$ : resolved shear stress vs. shear strain curves for the two active slip systems (solid and dotted lines). (b) Single slip $\left(\dot{\bar{\epsilon}}_{11}=\dot{\bar{\epsilon}}_{22}=0, \dot{\bar{\epsilon}}_{12}=\frac{1}{2} \dot{\gamma}\right.$ ): resolved shear stress vs. shear strain curves for the active slip system.

here, consistent with previous 2D DD simulations in the literature. These results are omitted for clarity.

Fig. 4(a) shows the simulated stress-strain behavior under symmetric double slip conditions for four different initial yield strengths $\tau_{y} \approx 30,35,50$ and $65 \mathrm{MPa}$. The lowest yield strength specimen corresponds to a crystal free of preexisting obstacles and containing an initial dislocation density of $\sim 50 \mu \mathrm{m}^{-2}$, while the higher strength specimens have a fixed population of initial obstacles with average spacing $L_{\text {obs }}=600,300$ and 150 $\mathrm{nm}$. Hence, strain hardening in the lowest strength specimen is entirely due to the formation of junction obstacles, while hardening in the higher strength specimens is due to the combined strengthening effect of preexisting obstacles and forest junctions. Fig. 4(a) shows the stress-strain response for both active slip systems, which are nearly identical due to the symmetric loading orientation. An approximately linear hardening response is predicted irrespective of the initial yield strength, indicating that the forest hardening mechanism is operative in addition to the strengthening due to the pre-existing fixed obstacles. The post-yield strain hardening rate is estimated using a linear least squares fit to the resolved shear stress vs. strain curves and, averaged over multiple realizations, yields $\mu / 70$ for $L_{\text {obs }}=600 \mathrm{~nm}$. This is somewhat larger than the experimental range $\mu / 200-\mu / 100$ but comparable; the effect of various model parameters on the predicted hardening rate will be discussed below.

Fig. 4(b) shows the stress-strain response of the same unit-cell subjected to single slip loading conditions $\left(\dot{\bar{\epsilon}}_{11}=\dot{\bar{\epsilon}}_{22}=0, \dot{\bar{\epsilon}}_{12}=\frac{1}{2} \dot{\gamma}\right)$. The response of the primary slip system shown in the figure exhibits very little strain hardening. The resolved shear stresses acting on the two secondary slip systems oriented at $\pm 60^{\circ}$ relative to the primary system (not shown) are approximately one half that of the primary system, for pure shear loading applied in the $\mathbf{e}_{1}-\mathbf{e}_{2}$ plane. This stress level is below the yield stress for the secondary systems and so there is no significant dislocation activity on these systems. Hence, the above results show that although a constant value of 10 is adopted for the parameter $K$ 


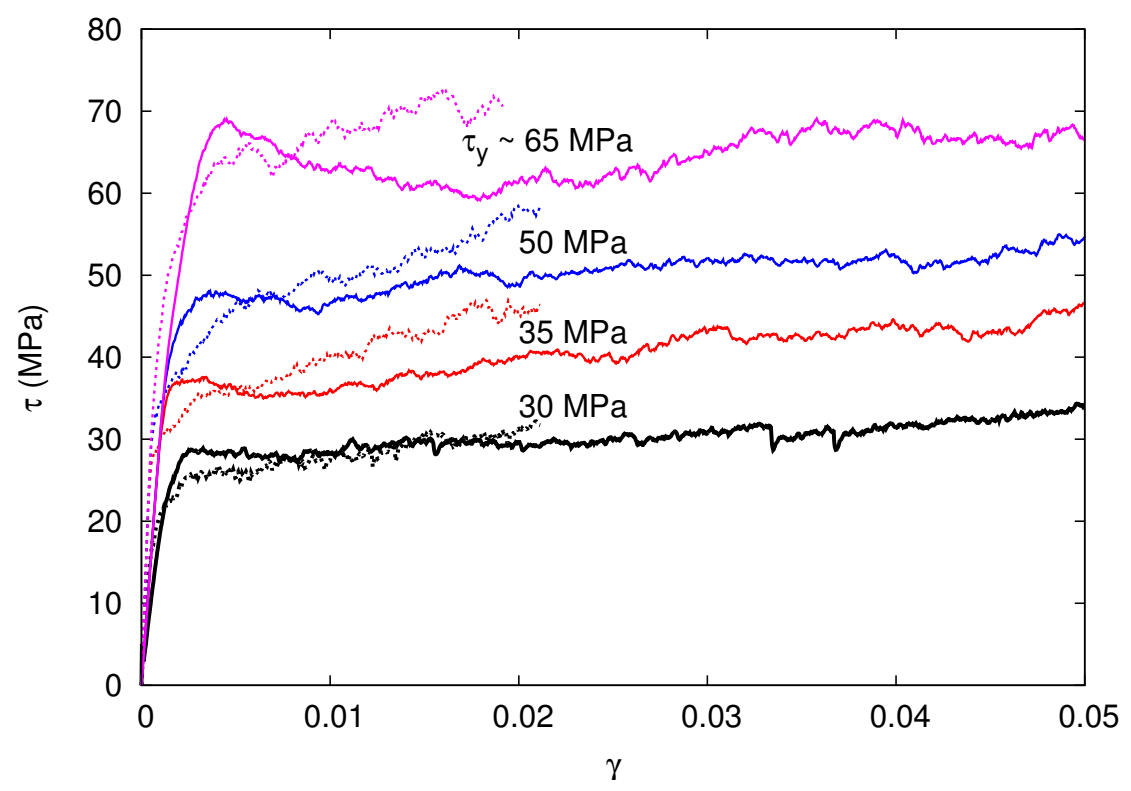

Figure 5: Resolved shear stress vs. shear strain curves for the primary (solid lines) and secondary (dotted lines) slip systems for crystals of various initial yield strengths $\tau_{y}$ under asymmetric double slip conditions $\left(\dot{\bar{\epsilon}}_{11}=-\dot{\bar{\epsilon}}_{22}=\dot{\bar{\epsilon}}_{12}\right)$.

in Eq.(5) irrespective of the loading orientation, the mean free path $L^{i}$ for the active slip system is much larger in single slip and the hardening rate is much smaller.

Fig. 5 shows the response of the primary and secondary slip systems in the case of the asymmetric double slip orientation corresponding to $\dot{\bar{\epsilon}}_{11}=\dot{\bar{\epsilon}}_{12}$. As expected, the strain hardening rate in the secondary system is higher than for the primary system, since the higher dislocation activity in the primary system leads to greater forest hardening for the dislocations on the secondary system. Fig. 6 shows the stress-strain curves under different loading orientations averaged over multiple simulations, to better show the differences in hardening rate among different loading conditions. As expected, the lowest hardening rate is obtained under single slip conditions, consistent with the self-hardening of a crystal during Stage I deformation. The strain hardening rate under symmetric double slip conditions is consistent with the athermal Stage II deformation. Under asymmetric double slip conditions, the primary slip system exhibits a lower hardening rate and the secondary system exhibits a higher hardening rate, as compared to those obtained under symmetric double slip conditions.

The hardening rate predicted by the new 2.5D DD method is a function of the two parameters in the constitutive rules: the junction strength parameter $\alpha$, Eq.(4), and the mean-free path parameter $K$, Eq.(5). In addition, the local density is evaluated in a neighborhood containing $N$ dislocations, and so the hardening rate should not depend on $N$. We now examine the role of these parameters on the material response under symmetric double slip conditions. Fig. 7 shows the stress-strain curves predicted for symmetric double slip for various values of $N$. The predicted hardening rate is nearly independent of $N$ in the range $N=5-100$. Values of $N<5$ make the sampling size too small for a reliable density calculation while large values of $N$ suppress local density 


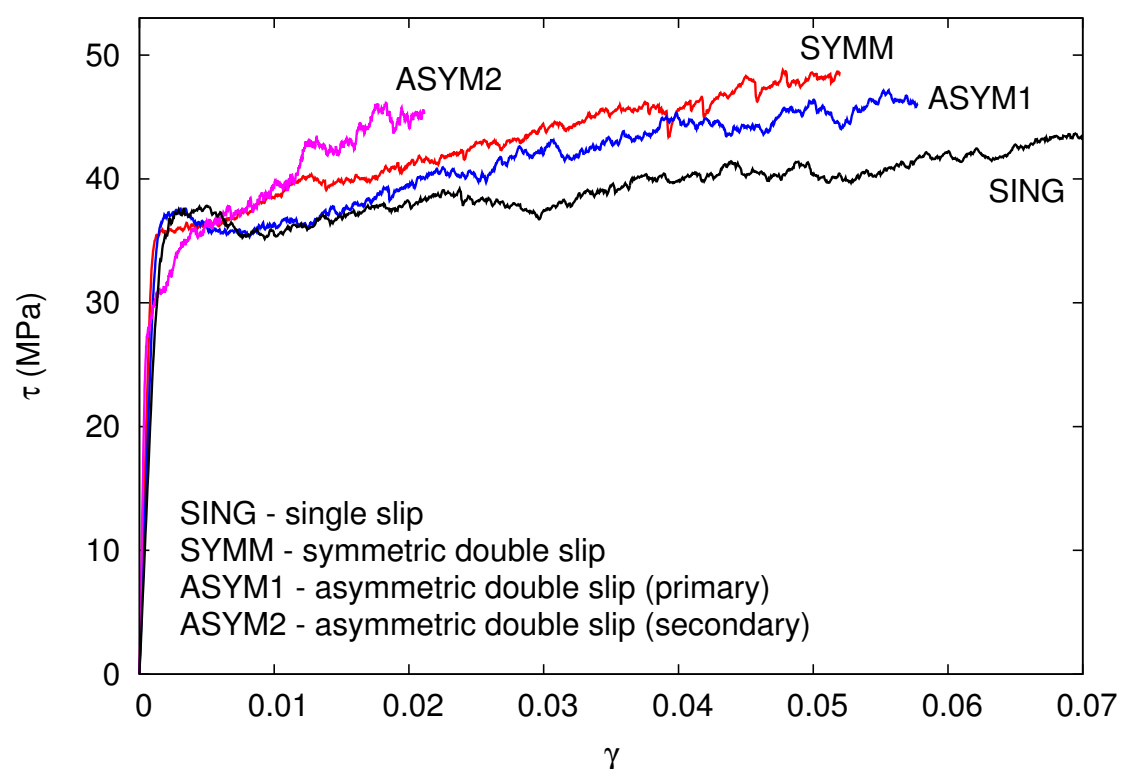

Figure 6: Resolved shear stress-strain curves averaged over multiple simulations under various loading orientations.

fluctuations. Fig. 7 shows that the strain hardening disappears for $N \geq 200$, which suggests an important role of local density fluctuations on the overall strain hardening rate.

Examination of the dislocation structures in the unit cell reveals that the microstructure at various stages of deformation consists of dense dislocation clusters separated by relatively dislocation free zones, as seen in Fig. 8(a) for one of the symmetric double slip simulations at $\sim 0.3 \%$ strain. As the plastic strain increases, initially dislocation free zones accumulate dislocations as observed in Fig. 8(b) for the same simulation at $\sim 1 \%$ strain, although the dislocation density remains highly non-uniform throughout the deformation. Although the cell like dislocation structures in Fig. 8 appear to indicate some sort of microstructuring or pattern formation within the RVE, the walls of these cells are invariably aligned with the orientation of the slip systems, indicating that these are dense dislocation pileups anchored by strong obstacles/junctions and not patterns formed due to some long range ordering. The results of Fig. 7 indicate that the formation of strong junctions in regions of high local dislocation density is probably responsible for the strain hardening effect, which disappears when the size of the sampling region becomes too large to capture these density fluctuations, i.e. $N \geq 200$. Further results below use $N=50$.

The effect of the mean free path parameter $K$ on the hardening rate is shown in Fig. 9. Fig. 9(a) shows the resolved shear stress vs. shear strain response under symmetric double slip and Fig. 9(b) shows the dislocation density, $\rho$, vs. strain. A wide range of values of $K$ is considered in Fig. 9, with a lower limit $K=1$ corresponding to $L^{i}=L_{f}^{i}$ in Eq.(5), i.e. a dislocation forming junctions with every forest dislocation on its glide path, and the upper limit $K=\infty$ corresponding to junction formation turned off completely. Note that no strain hardening is predicted in the latter case, consistent with previous results in the literature using the 2D model, while the predicted hardening rate is highest for $K=1$. 
This is a pre-print of the following article: Keralavarma, S. M.; Curtin, W. A. J. Mech. Phys. Solids 2016, 95, 132-146.. The formal publication is available at http://dx.doi.org/10.1016/j.jmps.2016.05.028

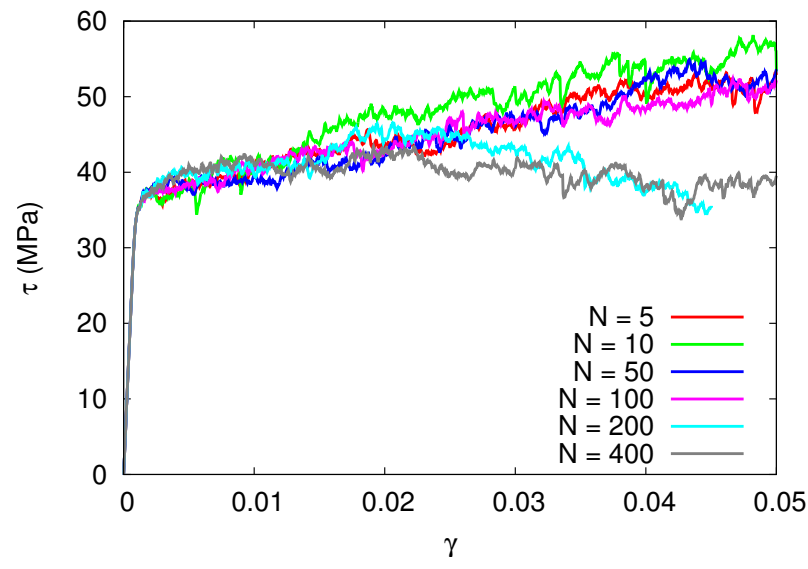

Figure 7: Effect of parameter $N$, which controls the size of the sampling neighborhood in the 2.5D algorithm, on the predicted hardening rates under symmetric double slip conditions.
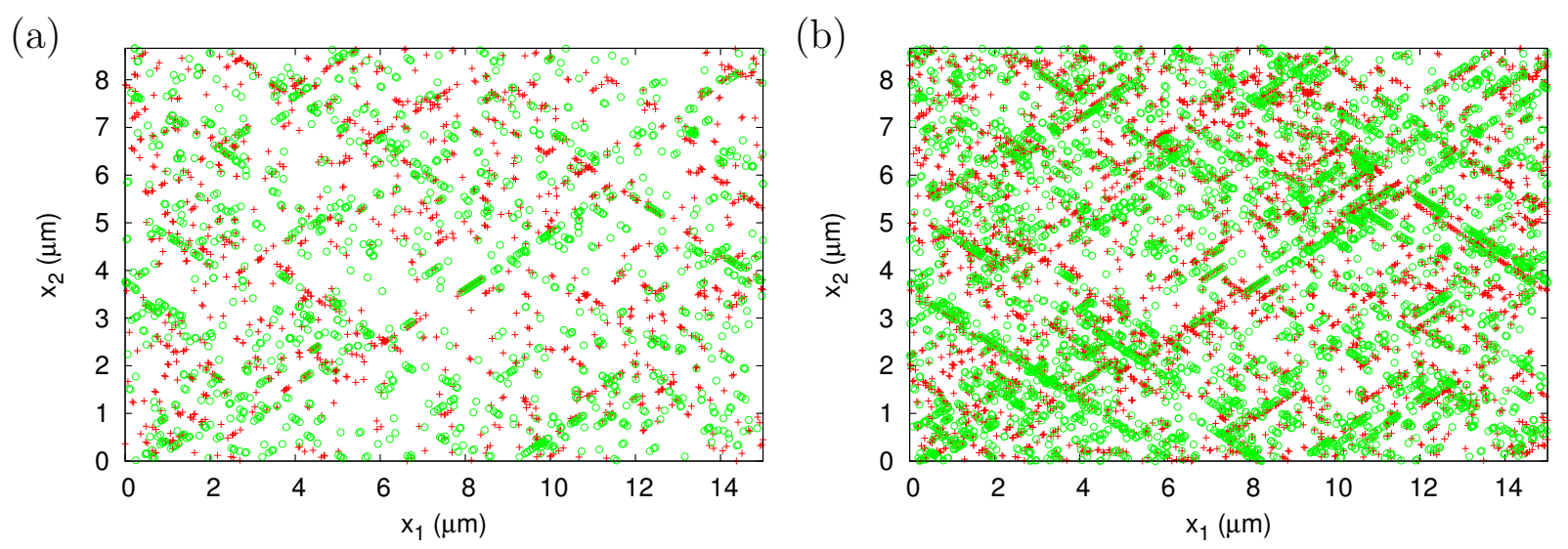

Figure 8: Dislocations structures at two different strain levels in a symmetric double slip simulation: (a) $\gamma \approx 0.3 \%$ and (b) $\gamma \approx 1 \%$. The red + symbols are positive dislocations and green open circles are negative dislocations.
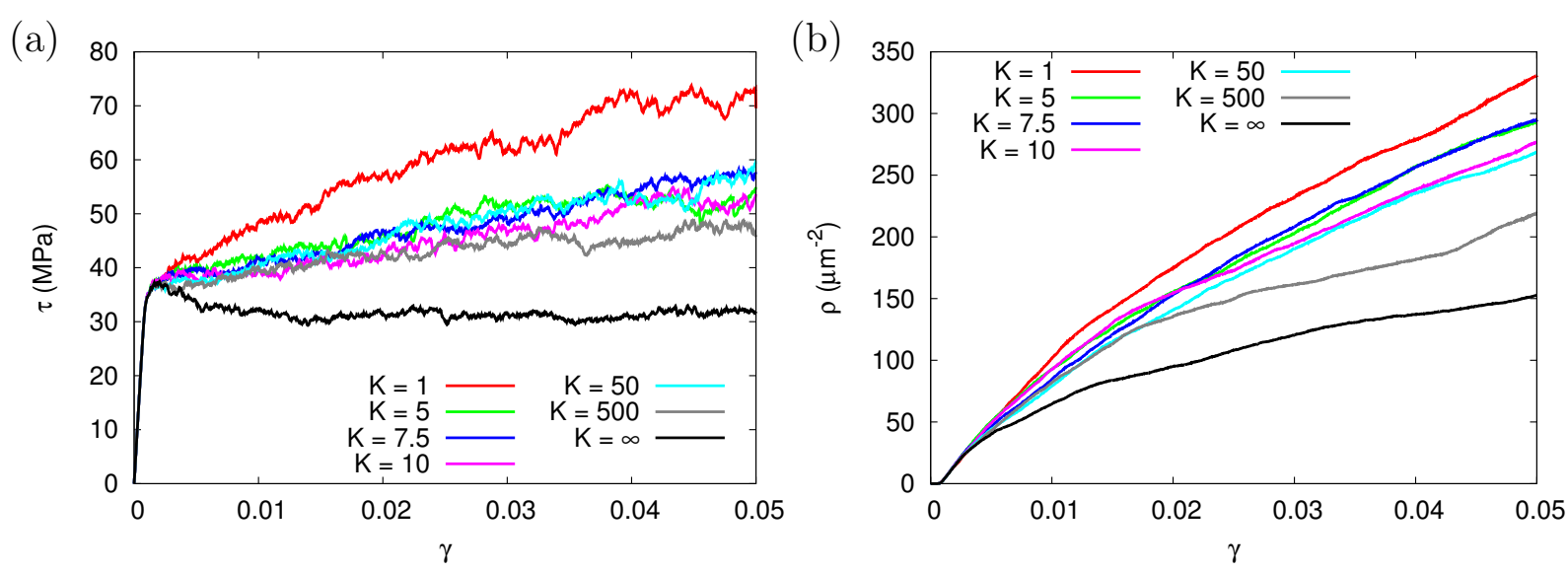

Figure 9: Effect of the mean free path parameter $K$ in Eq.(5) on the predicted hardening rates under symmetric double slip conditions: (a) resolved shear stress vs. shear strain and (b) dislocation density vs. shear strain. 

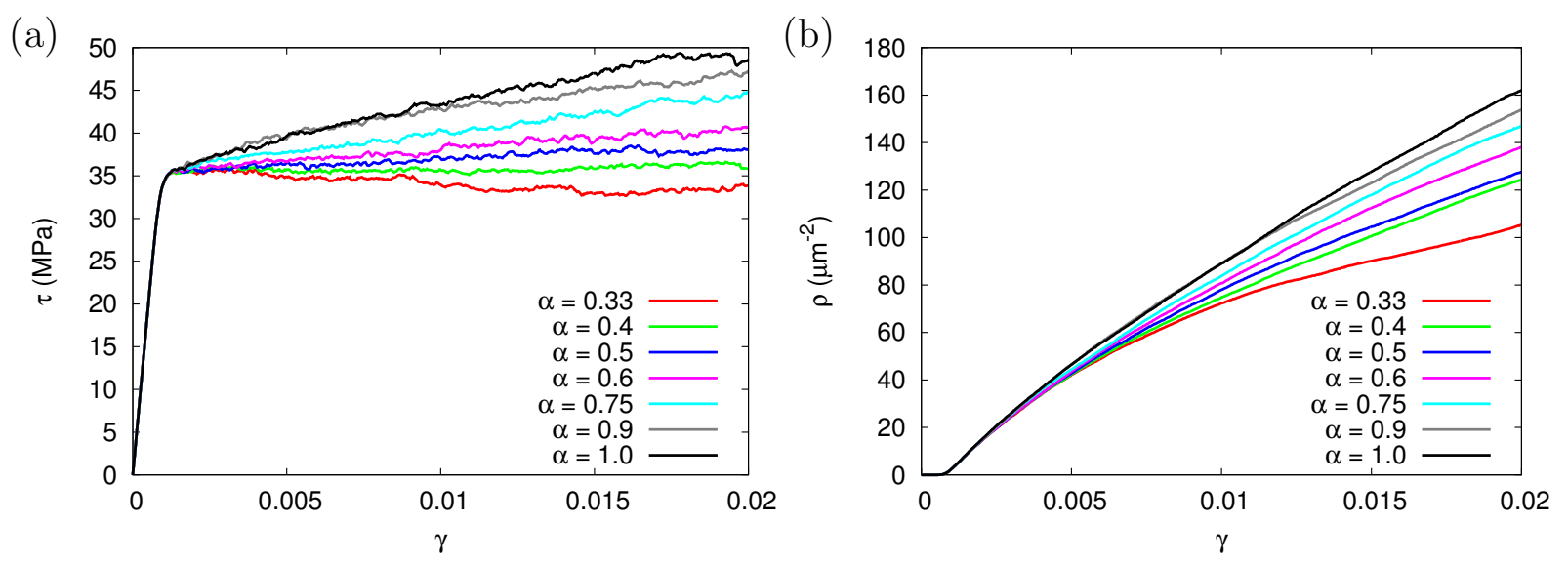

Figure 10: Effect of junction strength parameter $\alpha$ on the predicted hardening rates under symmetric double slip conditions: (a) resolved shear stress vs. shear strain and (b) dislocation density vs. shear strain.

The true mean free path of glide dislocations as a function of strain is difficult to estimate experimentally, and the value of $K$ can only be estimated indirectly from analysis of 3D DD simulation data. Approximate values of $K$ for various multi-slip loading directions in an FCC crystal, estimated using 3D simulations, fall in the range 5-10 (Devincre et al., 2008). Interestingly, our simulations show that within the range $K \approx 5-50$, the evolution of the flow stress and the dislocation density are relatively insensitive to the actual choice of $K$, thus justifying our use of a constant $K=10$ irrespective of the loading orientation. A related subtle point is the definition of the dislocation density $\rho$ in $2 \mathrm{D}$, which excludes the density of the junction obstacles formed by the 2.5D algorithm, although the dislocation line density in 3D does include a contribution from the junction segments. However, this cannot be accounted for in a $2 \mathrm{D}$ simulation without specification of an additional parameter relating to the ratio of the junction density to the overall dislocation density in 3D. Assuming this ratio to be approximately 30\%, as evidenced from 3D simulations (Devincre et al., 2008), it is observed that the estimated total dislocation densities increase from those shown in Fig. 9(b) by approximately 10-15\%, although the above observations regarding the effect of parameter $K$ remain unchanged.

The effect of the parameter $\alpha$, which determines the strength of the junction obstacles, is shown in Fig. 10 over the range $0.33-1$. The hardening rate, $\theta=\partial \tau / \partial \gamma$, increases monotonically with $\alpha$, with values of $\alpha \leq 0.5$ showing little strain hardening. The increase in hardening rate is correlated with a corresponding increase in the rate of dislocation density evolution, shown in Fig. 10(b). The normalized hardening rate $\theta / \mu$ is estimated using a linear least squares fit to the post yield stress-strain curve and averaged over multiple realizations of the simulations to account for statistical variations due to the initial source distribution. Fig. 11 shows $\theta / \mu$ versus $\alpha$ along with the experimentally-measured range of the athermal hardening rates in an FCC material (Argon, 2008). Despite significant scatter, a clear monotonic trend of hardening rate vs. $\alpha$ emerges, and the rate for $\alpha \sim 0.5-0.6$ is consistent with experiments. This range of values is also consistent with an isotropic line tension estimate for $\alpha$ as the average strength of possible junction types 


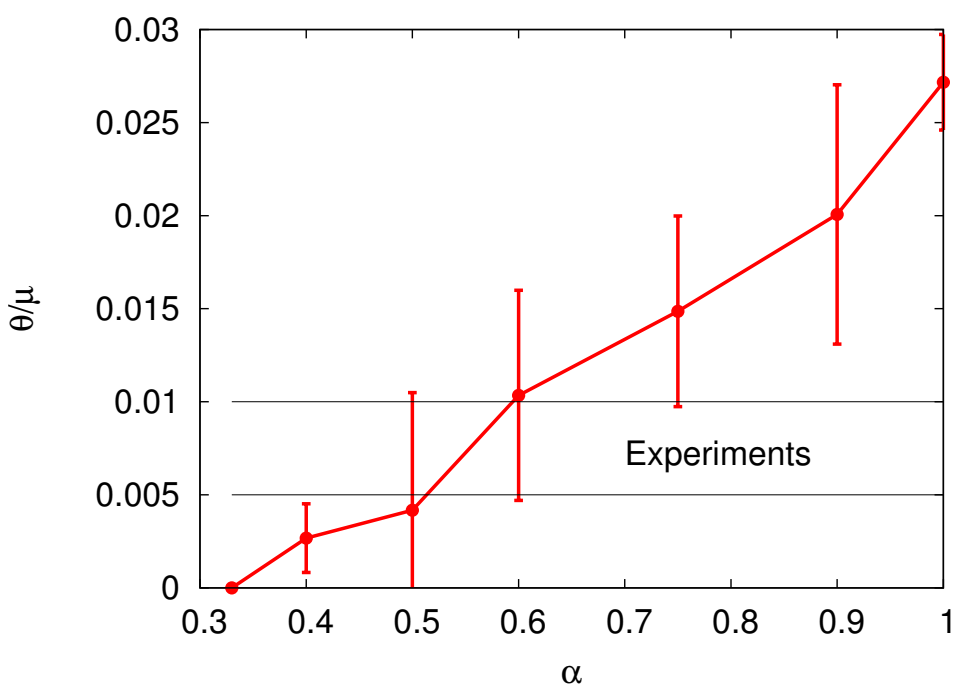

Figure 11: The predicted strain hardening rates $\theta$, normalized by the shear modulus $\mu$, as a function of the junction strength parameter $\alpha$. The horizontal band shows the experimentally observed range of the athermal hardening rates in FCC materials.

in an FCC crystal weighted by their frequency of occurrence.

Overall, the 2.5D DD algorithm presented here provides very good agreement in terms of differences in strain hardening with loading conditions, independence of the behavior to the parameter $N$ over a reasonable range, insensitivity to $K$ over its physical range, and realistic strain hardening rates for reasonable values of $\alpha$. Within the limits of small strain, there is no significant feature of the Stage I-II hardening in real single-crystal materials that is not captured well by the present 2.5D DD model.

\section{Discussion}

Phenomenological relations have long been used in continuum plasticity models to capture the evolution of flow stress with plastic deformation. The most well-known relationship is the Taylor hardening law, which states that the flow stress is proportional to the square root of the dislocation density. Along with the fact that the stress-strain curve is approximately linear in the early stages of plastic flow, when recovery processes are not active, this has been used to infer that the dislocation density evolves as the square of the accumulated plastic strain. However, this model does not account for the mutual hardening effect of one slip system on another in a single crystal, known as latent hardening. Building on experimental work from to 60s (Kocks and Brown, 1966; Kocks, 1970), Peirce et al. $(1982,1983)$ proposed a latent hardening model for use in continuum crystal plasticity simulations of the form

$$
\dot{\tau}^{(k)}=\sum_{l} h_{k l}\left|\dot{\gamma}^{(l)}\right|, \quad h_{k l}=q h+(1-q) h \delta_{k l}
$$

where $h$ is the self hardening rate of a slip system, $q$ is the ratio between the self hardening and latent hardening rates, and $\delta_{k l}$ is the Kronecker delta function. The value of $q$ is known 
to fall in the range 1-1.4, and a commonly used value is 1.4 (Peirce et al., 1983). The new 2.5D DD method has been shown to produce physically observed behavior under multiple slip conditions, and so can be used to estimate the value of the latent hardening ratio $q$. In a planar crystal with three slip systems as considered here, Eq.(17) predicts that the strain hardening rate $\theta^{(k)}=\partial \tau^{(k)} / \partial \gamma^{(k)}$ of the primary slip system is $(1+q r) h$ while that of the secondary system is $\left(1+\frac{q}{r}\right) h$, where $r \leq 1$ is the ratio of the slip rates on the secondary and primary slip systems. Thus, the value of $q$ may be determined by comparing the hardening rates between two active slip systems with unequal slip rates. Here, we use the average stress-strain curves in Fig. 6 for the asymmetric double slip simulation, corresponding to $r=0.366$. Estimating the slopes of the stress-strain curves for the primary and secondary slip systems and solving for the value of $q$ using the above equations yields $q \approx 1.27$, well within the accepted range for $q$. Together with the fact that our simulations predict a reasonable value for the absolute hardening rate under symmetric double slip conditions (and therefore the value of $h$ itself) as shown in Fig. 10(b), the 2.5D DD method captures the typical behavior in crystal plasticity.

Eq.(4) for the strength of junction obstacles in $2 \mathrm{D}$, with $L_{f}^{i}=1 / \sqrt{\rho_{f}^{i}}$, also hints at a generalized Taylor-type relationship between the slip system strengthening and the evolution of the forest dislocation density. However, it is noted that using Eq.(4) for the local junction strengths in terms of the local dislocation densities does not automatically imply a Taylor relationship between the average slip system strengths and dislocation densities. Hence it is interesting to investigate the relationship between the average flow stress and the forest dislocation density for each slip system. The existence of preexisting obstacles complicates the picture due to the fact that the resultant flow stress is a possibly non-linear combination of the strength due to preexisting obstacles, which is well predicted by Eq.(3), and that due to the junction obstacles. In the absence of preexisting sources and obstacles, the flow strength due to junction obstacles acting alone may be expected to follow a similar relationship as in Eq.(3), where $\tau_{\text {obs }}$ and $\tau_{\text {obs }}$ are replaced by the average strength and spacing of junction obstacles in the entire specimen. Using Eqs. (4) and (5) in (3), this yields

$$
\bar{\tau}_{f}^{i}=\bar{\alpha} \mu b \sqrt{\bar{\rho}_{\mathrm{jcn}}^{i}}
$$

where $\bar{\rho}_{\mathrm{jcn}}^{i}$ is the average forest dislocation density for slip system $i$ and $\bar{\alpha}$ is the Taylor hardening coefficient. When preexisting sources and obstacles are added, the combined strengthening due to both populations of obstacles could be expected to follow a relationship of the form (6), where $\tau_{1}=\tau_{y}$ from Eq.(3) and $\tau_{2}=\bar{\tau}_{f}^{i}$. In order to check this hypothesis, Fig. 12 shows the square of the flow stress $\tau$, normalized by the material shear modulus $\mu$, as a function of the forest dislocation density multiplied by the square of the Burgers vector $b$. The response of active slip systems under symmetric and asymmetric double slip are compared and, following an initial transient, an approximately linear scaling is obtained, indicating that the exponent in Eq.(6) is indeed close to 2. The slope of the curve is the Taylor hardening coefficient $\bar{\alpha}$, whose value is known to be of the order of unity and $\sim 1 / 3$ for random polycrystals. Calculation of the slopes from Fig. 12 yields a value in the range $\sim 0.4-0.5$, which is close to the expected value. Using an alternative definition of $\rho$ that also includes a $30 \%$ contribution from the junctions yields lower values 


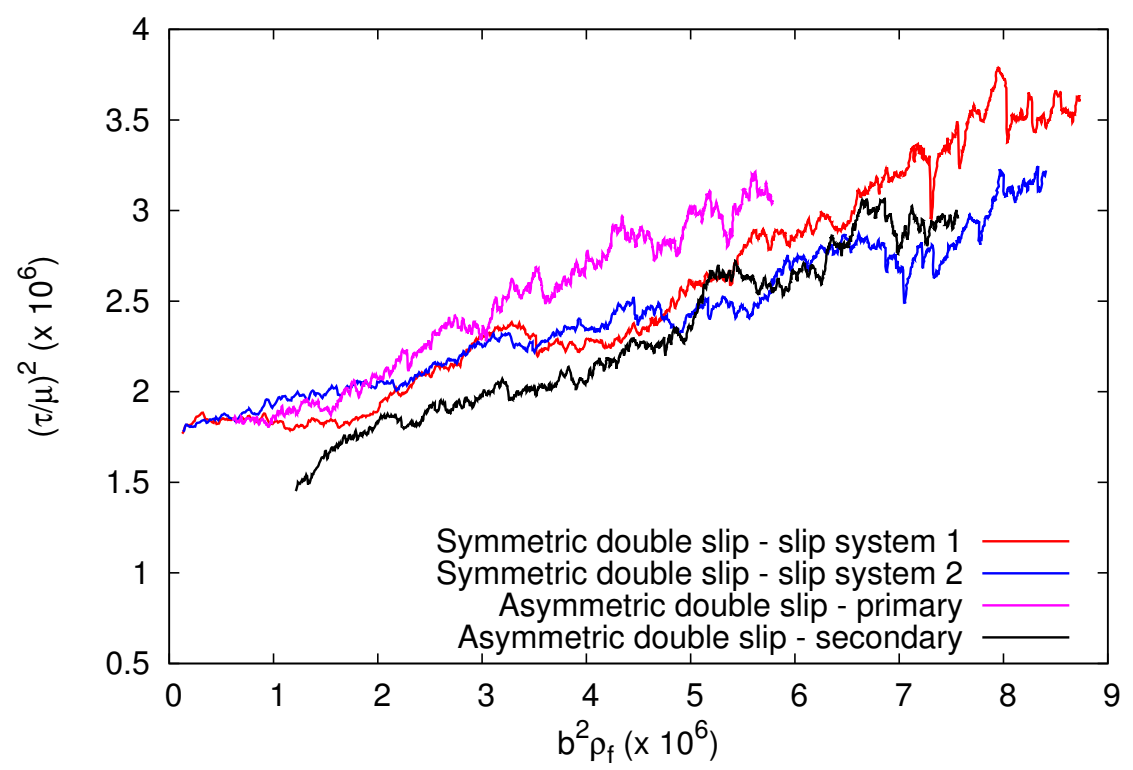

Figure 12: Scaling of the square of the flow strength as a function of the forest dislocation density for the active slip systems under multiple slip conditions. Appropriate non-dimensionalized quantities are plotted on both axes.

of $\bar{\alpha}$ in the range $0.35-0.45$. It is emphasized that although the Taylor form is suggested by our constitutive equations for strain hardening in section 2.2 , the emergence of this result is not obvious for the reasons cited above. The deviations from linearity in the early stages of plastic flow in Fig. 12 are indicative of the fact that the density of forest dislocations at this stage is much smaller than that of the preexisting obstacles and hence the exponent in (6) is probably different from 2.

\section{Conclusion}

Two dimensional discrete dislocation (2D DD) simulations are widely used to solve a variety of complex boundary value problems where full 3D DD is prohibitively expensive in terms of computing effort. However, the physics in 2D DD is unable to accommodate the $3 \mathrm{D}$ effects that cause strain hardening. To rectify this and create a new $2.5 \mathrm{D} \mathrm{DD}$ model that captures 3D effects, we have proposed a set of constitutive rules for dislocation interactions in 2D based on projecting 3D phenomena into 2D. The constitutive laws lead to a dynamic evolution of the dislocation mean free path, and to the emergence of overall strain hardening. The physically-motivated parameters in the new constitutive rules are obtained from 3D DD simulations, and so are well-bounded. The 2.5D DD model then predicts stress-strain behavior of bulk single crystals under homogeneous imposed deformation that exhibits several important effects of 3D dislocation plasticity. Specifically, the method captures both initial yielding and subsequent hardening, the differences between single and multiple slip, appropriate latent hardening under asymmetric multiple slip, and multiple slip hardening rates that are consistent with experiment. The model has an internal evolving length scale associated with the development of the forest dislocation network that may influence size-dependent plasticity. In the future, the new $2.5 \mathrm{D}$ 
DD method can be applied to problems such as fracture toughness and fatigue behavior under cyclic loading, where hardening phenomena are expected to play an important role.

\section{Acknowledgments}

Support for this work was partially provided through a European Research Council Advanced Grant, "Predictive Computational Metallurgy", ERC Grant agreement No. 339081 - PreCoMet and partially funded by EPFL through its support for the Laboratory for Multiscale Mechanics Modelling (LAMMM). SMK acknowledges partial support from the Department of Science and Technology, Government of India, under project number SB/FTP/ETA-0312/2013. 


\section{References}

Amodeo, R. J., Ghoniem, N. M., 1990. Dislocation dynamics. I. A proposed methodology for deformation micromechanics. Phys. Rev. B41, 6958-6967.

Argon, A. S., 2008. Strengthening Mechanisms in Crystal Plasticity. Oxford University Press, USA.

Arzt, E., 1998. Size effects in materials due to microstructural and dimensional constraints: A comparative review. Acta Mater. 46 (16), 5611-5626.

Benzerga, A. A., Bréchet, Y., Needleman, A., Van der Giessen, E., 2004. Incorporating three-dimensional mechanisms into two-dimensional dislocation dynamics. Model. Simul. Mater. Sci. Eng. 12, 159-196.

Brown, L. M., Ham, R. K., 1971. Dislocation-particle interactions. Strengthening methods in crystals, 9-135.

Chakravarthy, S., Curtin, W., 2011a. New algorithms for discrete dislocation modeling of fracture. Model. Simul. Mater. Sci. Eng. 19, 045009.

Chakravarthy, S. S., Curtin, W. A., 2010a. Effect of source and obstacle strengths on yield stress: A discrete dislocation study. J. Mech. Phys. Solids 58, 625-635.

Chakravarthy, S. S., Curtin, W. A., 2010b. Origin of plasticity length-scale effects in fracture. Phys. Rev. Lett. 105, 115502.

Chakravarthy, S. S., Curtin, W. A., 2011b. Stress-gradient plasticity. Proceedings of the National Academy of Sciences 108, 15716-15720.

Chng, A. C., O’Day, M. P., Curtin, W. A., Tay, A. A. O., Lim, K. M., 2006. Fracture in confined thin films: a discrete dislocation study. Acta Mater. 54 (4), 1017-1027.

De Borst, R., Sluys, L. J., Muhlhaus, H.-B., Pamin, J., 1993. Fundamental issues in finite element analyses of localization of deformation. Engineering computations 10, 99-121.

Deshpande, V. S., Needleman, A., Van der Giessen, E., 2001. A discrete dislocation analysis of near-threshold fatigue crack growth. Acta Mater. 49, 3189.

Deshpande, V. S., Needleman, A., Van der Giessen, E., 2004. Discrete dislocation plasticity analysis of static friction. Acta Mater. 52 (10), 3135-3149.

Devincre, B., Hoc, T., Kubin, L., 2008. Dislocation mean free paths and strain hardening of crystals. Science 320, 1745-1748.

Dong, Y., Nogaret, T., Curtin, W. A., 2010. Scaling of dislocation strengthening by multiple obstacle types. Metallurgical and Materials Transactions A 41, 1954-1960.

Fleck, N. A., Hutchinson, J. W., 1997. Strain gradient plasticity. Adv. Appl. Mech. 33, $295-361$. 
Fleck, N. A., Muller, G. M., Ashby, M. F., Hutchinson, J. W., 1994. Strain gradient plasticity - theory and experiment. Acta Metall. Mater. 42, 475-487.

Foreman, A. J. E., Makin, M. J., 1967. Dislocation movement through random arrays of obstacles. Canadian Journal of Physics 45 (2), 511-517.

Gao, H., Huang, Y., Nix, W. D., Hutchinson, J. W., 1999. Mechanism-based strain gradient plasticity - I. Theory. J. Mech. Phys. Solids 47, 1239-1263.

Gómez-Garcia, D., Devincre, B., Kubin, L. P., 2006. Dislocation patterns and the similitude principle: 2.5d mesoscale simulations. Phys. Rev. Lett. 96, 125503.

Guruprasad, P. J., Benzerga, A. A., 2008. Size effects under homogeneous deformation of single crystals: A discrete dislocation analysis. J. Mech. Phys. Solids 56, 132-156.

Hirth, J. P., Lothe, J., 1968. Theory of Dislocations. Wiley, New York.

Hussein, M., Borg, U., Niordson, C., Deshpande, V., 2008. Plasticity size effects in voided crystals. J. Mech. Phys. Solids 56, 114-131.

Kocks, U. F., 1970. The relation between polycrystal deformation and single-crystal deformation. Metallurgical and Materials Transactions 1 (5), 1121-1143.

Kocks, U. F., Brown, T. J., 1966. Latent hardening in aluminum. Acta Metall. 14 (2), $87-98$.

Kocks, U. F., Mecking, H., 2003. Physics and phenomenology of strain hardening: the FCC case. Prog. Mater. Sci. 48, 171-273.

Kubin, L., Devincre, B., Hoc, T., 2008. Modeling dislocation storage rates and mean free paths in face-centered cubic crystals. Acta Mater. 56, 6040-6049.

Nicola, L., Xiang, Y., Vlassak, J. J., Van der Giessen, E., Needleman, A., 2006. Plastic deformation of freestanding thin films: experiments and modeling. J. Mech. Phys. Solids 54 (10), 2089-2110.

O’Day, M. P., Curtin, W. A., 2005. Bimaterial interface fracture: a discrete dislocation model. J. Mech. Phys. Solids 53 (2), 359-382.

Olarnrithinun, S., Chakravarthy, S. S., Curtin, W. A., 2013. Discrete dislocation modeling of fracture in plastically anisotropic metals. J. Mech. Phys. Solids 61 (6), 1391-1406.

Olmsted, D., HectorJr, L., Curtin, W., Clifton, R., 2005. Atomistic simulations of dislocation mobility in $\mathrm{Al}, \mathrm{Ni}$ and $\mathrm{Al} / \mathrm{Mg}$ alloys. Model. Simul. Mater. Sci. Eng. 13 (3), $371-388$.

Peirce, D., Asaro, R. J., Needleman, A., 1982. An analysis of non uniform and localized deformation in ductile single crystals. Acta Metall. 31, 1087-1119. 
Peirce, D., Asaro, R. J., Needleman, A., 1983. Material rate dependence and localized deformation in crystalline solids. Acta Metall. 31 (12), 1951-1976.

Segurado, J., Llorca, J., 2009. An analysis of the size effect on void growth in single crystals using discrete dislocation dynamics. Acta Mater. 57, 1427-1436.

Tvergaard, V., Hutchinson, J., 1992. The relation between crack growth resistance and fracture process parameters in elastic-plastic solids. J. Mech. Phys. Solids 40, 13771397.

Uchic, M. D., Shade, P. A., Dimiduk, D. M., 2009. Plasticity of micrometer-scale single crystals in compression. Annual Review of Materials Research 39, 361-386.

Van der Giessen, E., Needleman, A., 1995. Discrete dislocation plasticity: a simple planar model. Model. Simul. Mater. Sci. Eng. 3, 689-735.

Widjaja, A., Needleman, A., Van der Giessen, E., 2007. The effect of indenter shape on sub-micron indentation according to discrete dislocation plasticity. Model. Simul. Mater. Sci. Eng. 15 (1), S121-S131. 